\title{
LIQUEFACTION POTENTIAL AND PIEZOCONE RESPONSE
}

\author{
R.A. Vreugdenhil1,2, R.O. Davis' \& J.B. Berrill1,3
}

\begin{abstract}
A simple approximate analysis is presented for interpretation of cone penetration results when the cone resistance is affected by layering of soils with different stiffnesses. It is argued that the cone resistance senses the presence of a nearby layer elastically, and an approximate elastic analysis is developed to quantify the effect. Good comparisons with calibration chamber experimental results are found. This has important implications for soil classification and for assessment of liquefaction potential from cone penetration results.
\end{abstract}

\section{INTRODUCTION}

Often when comparisons are drawn between cone penetration tests (CPT) and standard penetration tests (SPT), an advantage is attributed to the CPT due to the continuous nature of the test results. The CPT gives a continuous measure of penetration resistance while the SPT can yield only a mean resistance over a soil depth of $30 \mathrm{~cm}$. SPT results cannot therefore adequately define thin layers or lenses of soil which in certain circumstances may be quite important.

In fact, the CPT itself may not precisely define thin soil layers, even though a continuous measure of penetration resistance is obtained. Many workers in the field have recognized that CPT resistance may be significantly affected by soil layering.

As the cone approaches a stiffer layer for example, it will "sense" the presence of this layer some distance before actual penetration of the stiffer soil occurs. Also, after the cone enters the stiffer layer, its response will continue to sense the softer soil above, and this will result in a lower resistance for some distance in the stiffer layer. These effects have been well documented in calibration chamber experiments (Canou [1], Foray and Pautre [2]).

Now consider the case where the cone approaches a thin layer, as illustrated in Figure 1. The cone will sense the layer as it draws nearer. However, since the layer is thin the resistance will also be affected by the second stiffness change at the bottom of the thin layer. When penetration of the thin layer occurs, the CPT resistance will be affected by both the soil above the layer and the soil below. For quite thin layers, the penetration resistance may be greatly different from the value obtained in a homogeneous deposit of the same soil.

\footnotetext{
1 Department of Civil Engineering, University of Canterbury

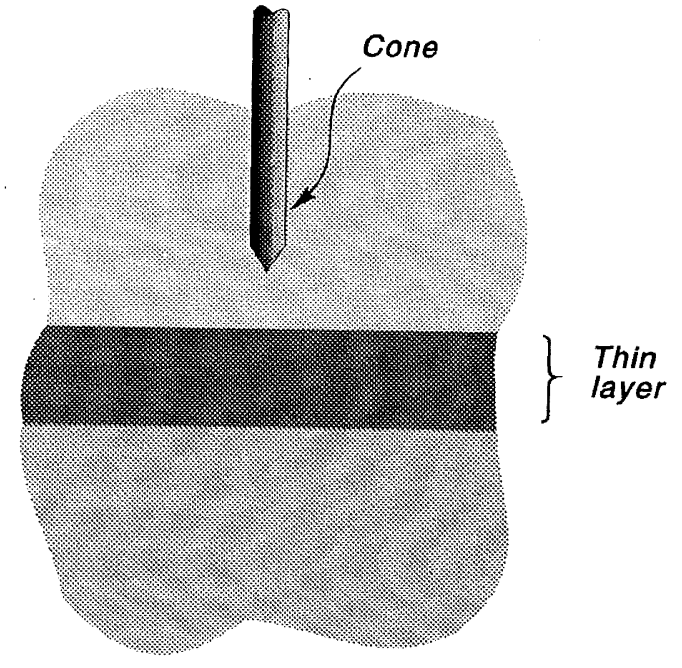

FIGURE 1. Penetrating cone approaching a layer of dissimilar soil.

In this paper an attempt is made to model the effects of layering by way of an elastic analysis. At first glance, elasticity may appear to be an extremely poor model for a penetration problem in which large plastic strains must occur near the cone point. However, it is not the actual penetration process that is being modeled, but the effect on the cone resistance of nearby layers of soil. This suggests that an elastic analysis may prove to be useful since the layering effect at a distance from the cone tip must be essentially elastic in nature. Moreover, the analysis produces results which compare favourably with calibration chamber test results. In fact, there may be potential application of the method to the interpretation of calibration chamber tests as well as to the interpretation of actual field CPT data. 


\section{ANALYSIS}

Consider two linearly elastic, incompressible half-spaces in bonded contact as shown in Figure 2(a). The CPT will be represented by a disc-shaped region of radius $a$ which supports a uniform applied stress $p_{o}$ as shown in the figure. Let $a$ be the radius of the cone, and let $\delta$ denote the vertical deflection at the centre of the loaded region.

The exact solution to this problem may be obtained by integrating the point-load solution of Plevako [3], but it is more convenient to employ an approximate analysis based on the Boussinesq half-space, point-load solution. The reason for employing the approximate rather than the exact solution lies in the need to represent multiple layering, for which Plevako's analysis cannot be used, while the approximate solution can. The assumption of incompressibility is based on the expectation that the effects at a distance which are being modeled here will result primarily from undrained soil deformations (there is no difficulty in modifying the analysis to encompass compressible elastic materials)

The first step in the analysis is to decompose the layered infinite space in Figure 2(a) into two elastic half-spaces as shown in Figure 2(b). Each half-space supports a uniform stress, $p_{o}^{\prime}$ or $p_{o}^{\prime \prime}$, over the disc-shaped region of radius $a$. The upper half-space is homogeneous, while the lower half-space is layered. For the upper half-space, Boussinesq's point-load solution may be easily integrated over the disc-shaped region to give the displacement $\delta^{\prime}$ at the centre of the disc. For an incompressible material we find

$$
\delta^{\prime}=\frac{p_{o}^{\prime} a}{2 G_{1}}
$$

Here $G_{1}$ denotes the elastic shear modulus of material 1. For the lower, layered half-space, an approximation can be used, based on Boussinesq's solution, in which the relative displacements in the two layers are combined, as proposed by

Poulos [4]. The displacement $\delta^{\prime \prime}$ at the centre of the loaded region is given by

$$
\begin{aligned}
\delta^{\prime \prime}=\frac{p_{o}^{\prime \prime} a}{2 G_{1}} & -\frac{p_{o}^{\prime \prime}}{2 G_{1}} \frac{a^{2}}{\sqrt{a^{2}+h^{2}}} \\
& +\frac{p_{o}^{\prime \prime}}{2 G_{2}} \frac{a^{2}}{\sqrt{a^{2}+h^{2}}}
\end{aligned}
$$

The three terms on the right hand side of this equation represent (i) the surface displacement of a homogeneous half-space composed of material 1, (ii) the displacement at depth $h$ beneath the centre of the loaded disc in a. homogeneous half-space composed of material 1 , and (iii) the displacement at depth $h$ beneath the centre of the loaded disc in a homogeneous halfspace composed of material 2. The difference between terms (i) and (ii) represents the relative shortening in the layer of thickness $h$. Term (iii) represents the displacement of a halfspace of material 2 below the depth $h$. Equation (2) is a well known approximation for the surface displacement of a layered half-space. It compares favourably with exact solutions based on Burmister's analysis [4].

Now the two half-space solutions in Figure 2(b) must be combined to represent the infinite space problem in Figure 2(a) This is accomplished applying the conditions of compatibility of
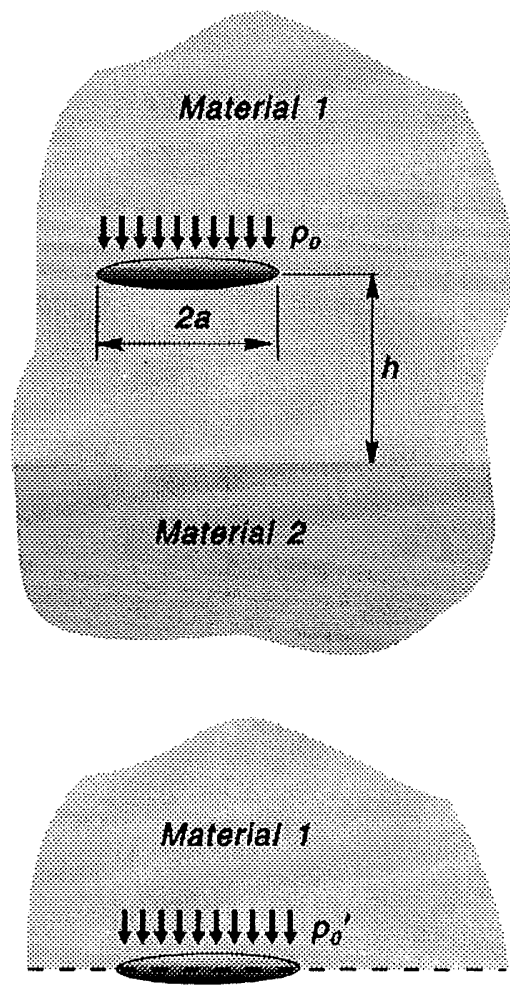

(b)

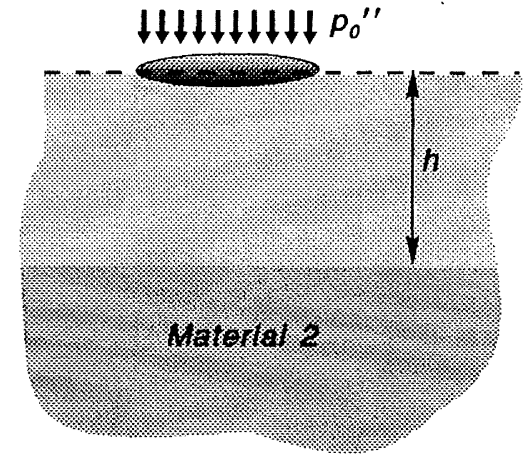

FIGURE 2. Method of analysis: (a) Representation of CPT by circular uniform load. (b) Decomposition of (a) into two halfspace problems.

displacements $\left(\delta^{\prime}=\delta^{\prime \prime}=\delta\right)$ and equilibrium $\left(p_{o}^{\prime}+p_{o}^{\prime \prime}=p_{o}\right)$ to obtain

$$
\delta=\frac{p_{o} a}{2 G_{1}}\left(\frac{1-\lambda_{1}}{2-\lambda_{1}}\right)
$$

where the general form for $\lambda$ is given by

$$
\lambda_{i}=\left(1-\frac{G_{i}}{G_{i+1}}\right) \frac{a}{\sqrt{a^{2}+h_{i}^{2}}}
$$

In effect, the two half-spaces are being joined on the horizontal plane passing through the loaded region. This approach is based on the fact that, for an incompressible material, Kelvin's solution for a point load in an infinite space gives exactly the same stresses and displacements in the half-space above or below the load point as Boussinesq's solution for a half-space with a point load equal to half that used in Kelvin's problem. 
The approximate solution gives results very close to Plevako's exact solution for this single layer geometry, and it becomes exact in the limiting cases where $h \rightarrow \infty$ or $h \rightarrow 0$.

It is an easy matter to generalize this analysis to multi-layered situations. In this way the displacement $\delta$ can be estimated at any point in a multi-layered infinite space. However, it will be convenient to recast the development in dimensionless form. The dimensionless parameter $\lambda_{i}$ has already been introduced in equation (4). Two additional parameters are defined as follows, the dimensionless stiffness ratio:

$$
k_{i}=G_{i+1} / G_{1}
$$

and the dimensionless penetration resistance:

$$
\eta=\frac{p_{o} a}{G_{1} \delta}
$$

Thus for any number of layers, $\eta$ may be defined by a functional relationship of the form

$$
\eta=F\left(\lambda_{1}, \lambda_{2}, \ldots, k_{1}, k_{2}, \ldots\right)
$$

The exact form of $F$ depends on the number of layers involved and the position at which the load is applied. For example, in the simple single layer case of Figure 2(a), for load points above the layer interface

$$
\eta=2\left(\frac{2-\lambda_{1}}{1-\lambda_{1}}\right) \quad \text { for } h>0
$$

while for load points below the layer interface

$$
\eta=2 k_{1}\left(\frac{2+k_{1} \lambda_{1}}{1+k_{1} \lambda_{1}}\right) \text { for } h \leq 0
$$

Now using equations (8) and (9) the dimensionless penetration resistance $\eta$ may be plotted as a function of position. Note the behaviour of $\eta$ at points distant from the interface, and at the interface itself

$$
\begin{aligned}
& \text { for } h / a \rightarrow \infty, \quad \eta \rightarrow 4 \\
& \text { for } h / a=0, \quad \eta=2\left(1+k_{1}\right) \\
& \text { for } h / a \rightarrow-\infty, \quad \eta \rightarrow 4 k_{1}
\end{aligned}
$$

It is apparent that $\eta$ will move smoothly through the interface, varying between a value of 4 in material 1 and a value of $4 k_{1}$ in material 2. Note also that at the interface the value of $\eta$ is exact. For more complex layering, equations (8) and (9) must be replaced by more complex expressions, but this is a straightforward matter.

In what follows $\eta$ will be used to model actual penetration resistance as a function of depth. This does not suggest that the elastic analysis given here accounts in any way for the immediate effects of penetration and plastic deformation which occur near the tip of a penetrating cone. The analysis is intended solely to model the effects of layering sensed by the cone in its own vicinity. To accomplish this, when actual values of cone resistance, $q_{c}$, are considered, they will be made dimensionless in such a way that they will agree with the value of $\eta$ when there is no layering in the near vicinity. Referring to the individual terms on the right hand side of equation (6), we assume $p_{o}$ and $a$ correspond directly to the measured penetration resistance $q_{c}$ and the cone radius. The quantity $G_{1} \delta$ must be set equal to a constant reference value which is chosen so that $\eta$ in the reference layer (material 1 ) tends to 4 at large distances from the layer 1 - layer 2 interface, that is

$$
G_{1} \delta=\frac{1}{4} \bar{q}_{c} a
$$

Here $\bar{q}_{c}$ is the mean value of measured penetration resistance in layer 1 . In one sense this calibrates the model to the actual data. In another sense it completely avoids the problems associated with the actual penetration process and focuses attention on the elastic effects of layering

\section{COMPARISON WITH EXPERIMENTAL DATA}

A number of calibration chamber experiments have been carried out using layered soil profiles by Canou [1] at CERMES, Paris, and by Foray [2] and co-workers at the IMG, Grenoble. The CERMES tests employed a mini-cone of diameter $11.3 \mathrm{~mm}$; the calibration chamber having dimensions of $180 \mathrm{~mm}$ diameter and $400 \mathrm{~mm}$ depth. In the Grenoble tests, a Parez cone of diameter $45 \mathrm{~mm}$ was used in a chamber measuring $1.20 \mathrm{~m}$ in diameter and $1.50 \mathrm{~m}$ in depth

Figures 3(a), 3(b) and 4 show dimensionless mini-cone resistance data (solid lines) and the corresponding theoretical resistance curves $\eta$ for two calibration chamber tests. In each case the actual mini-cone resistance is made dimensionless in such a way that its average value in the upper layer away from the interface is equal to 4. Vertical dimensions are shown measured from the upper soil surface in the chamber, and are made dimensionless by dividing by $a$.

Figures 3(a) and 3(b) show results from an experiment involving a soft layer over a stiffer layer. The experimental data (solid lines) in both figures are the same, but the theoretical results (dashed lines) are different. The theoretical dashed line on Figure 3(a) was obtained using equations (8) and (9), with $k_{1}$ set to 4.29. Calculations were performed without attempting to represent the chamber base, and consequently the experimental and theoretical curves bear only a reasonable likeness. In Figure 3(b) the base was included in the theoretical calculation by introducing a near-rigid layer at dimensionless depth 70.9 . Figure 3 (b) appears to model the experimental data remarkably well and the effect of the chamber base is seen to extend a significant distance upward into the sample.

Figure 4 shows an experimental plot in which three soil layers were involved: a soft layer sandwiched between two stiffer layers. The stiffness ratios used to model these results were $k_{1}=0.34$ and $k_{2}=2.51$. A rigid base layer was included in the calculation also.

Clearly, the theoretical results shown here do not agree exactly with the experimental data. Nevertheless, the theoretical penetration resistance appears to adequately model the layer effects observed in the experiments, and there is a remarkable degree of correlation despite the noisiness evident in the experimental traces.

Finally, it is evident from the figures that a soft layer requires only a relatively short distance of penetration for cone resistance to reach a steady state value. This seems reasonable since a soft layer would be expected to isolate the penetrating cone from materials it has yet to encounter, while a stiff layer will not have this effect. 


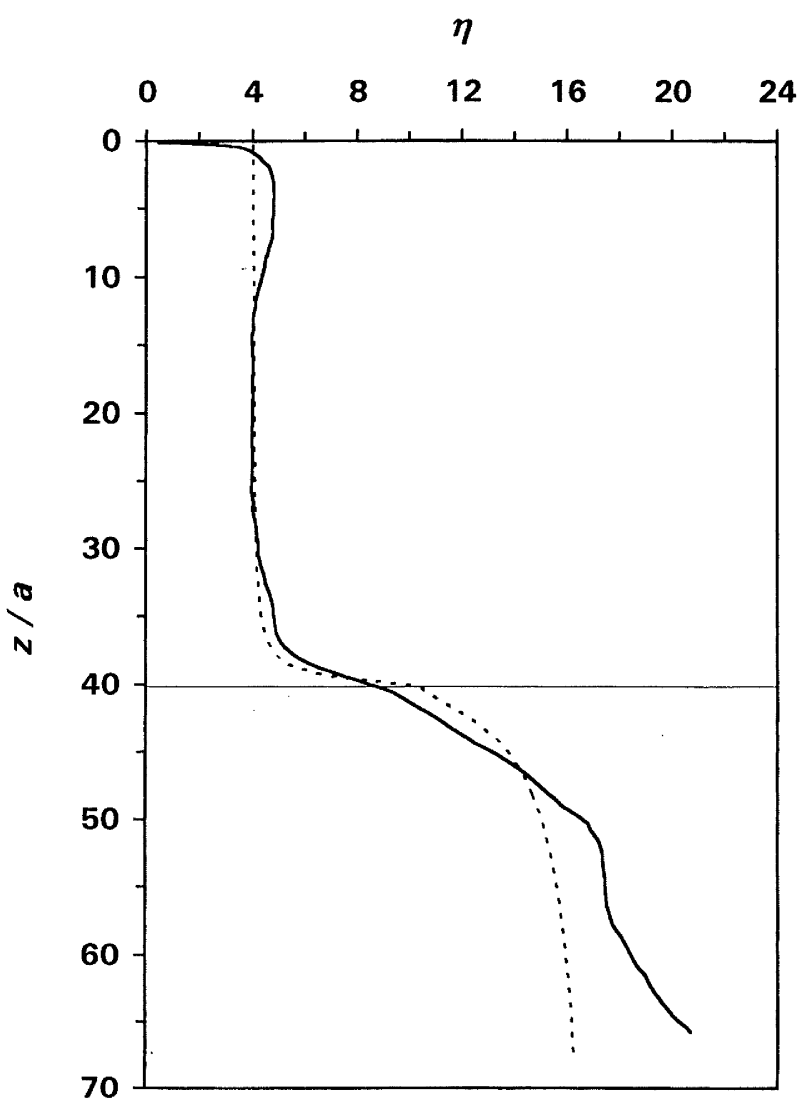

FIGURE 3(a). Comparison of theory with calibration chamber results (without chamber base).

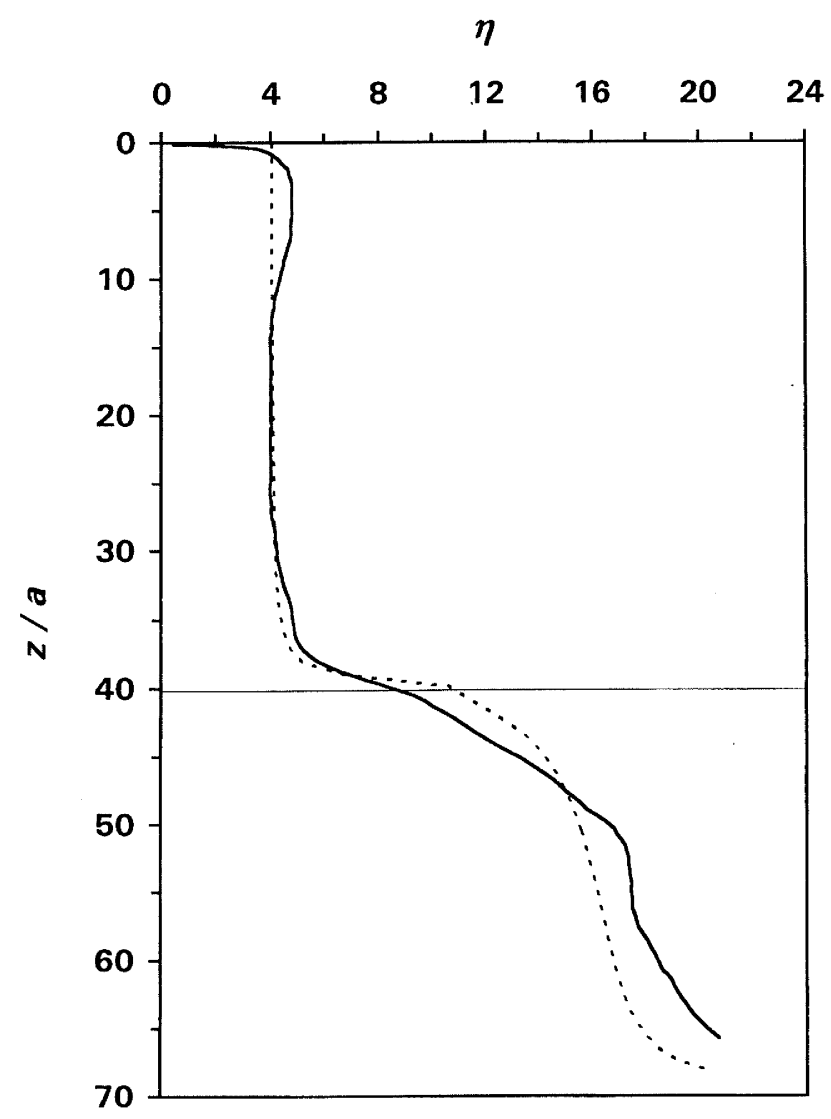

FIGURE 3(b). Comparison of theory with calibration chamber results (with chamber base).

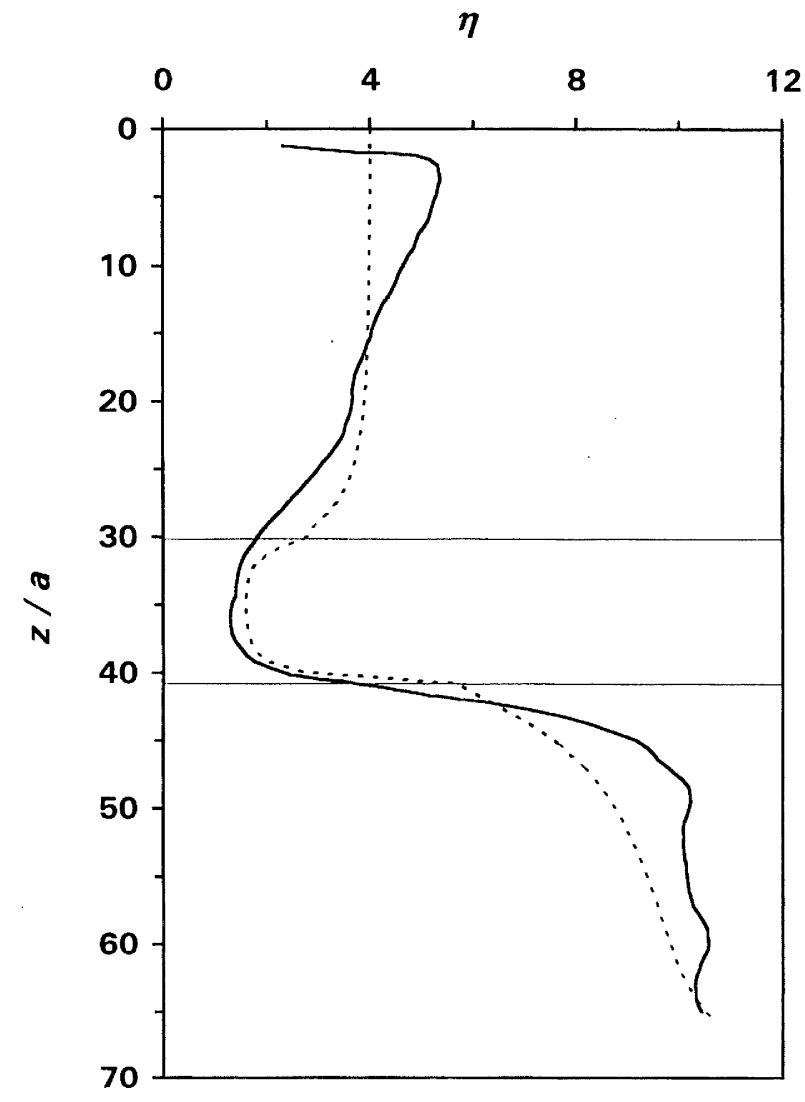

FIGURE 4. Comparison of theory with calibration chamber results. Three layer example.

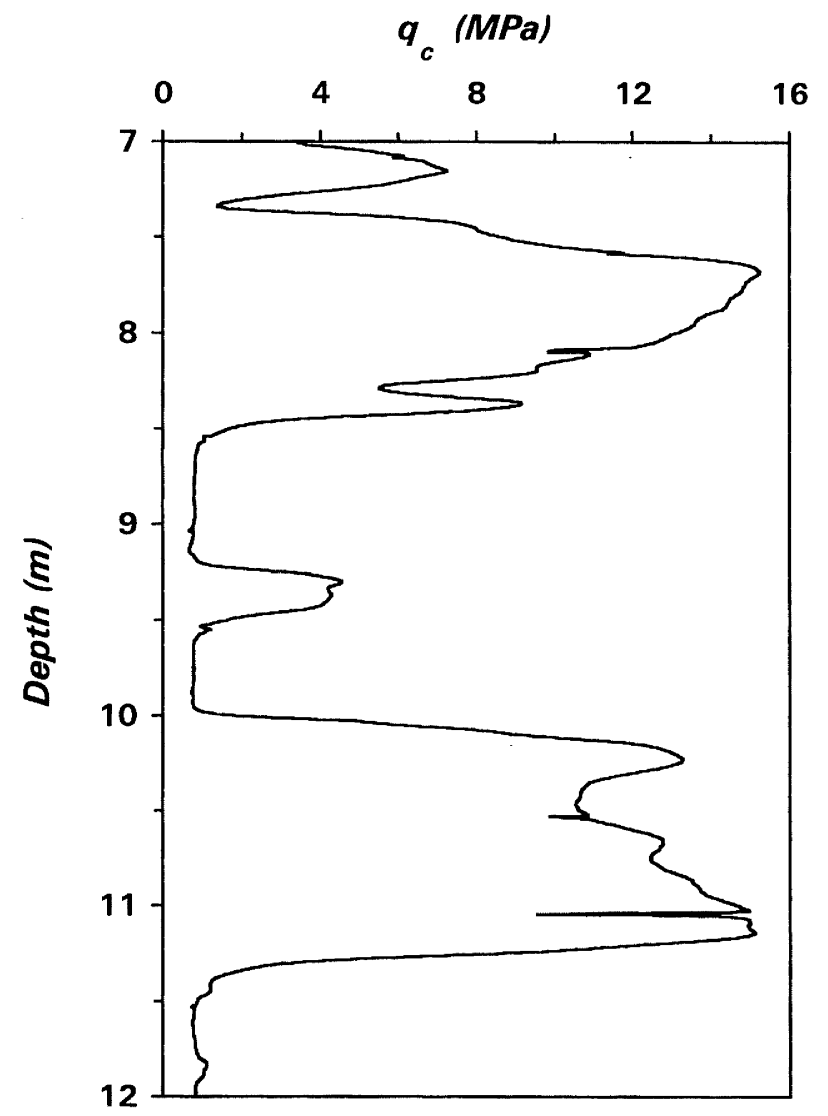

FIGURE 5. Measured penetration resistance at a site near the Salinas River in California. 


\section{APPLICATIONS}

The most obvious use for this analysis appears to be in the interpretation of cone resistance data where thin soil layers are involved. As an example of how a thin soil layer may be misclassified, consider the following actual field record. Figure 5 shows a section of a piezocone (CPTU) penetration resistance record obtained at a site near the Salinas River mouth in central California. There appears to be a thin layer of stiff soil embedded between two softer layers, within the segment between $8.5 \mathrm{~m}$ and $10 \mathrm{~m}$ depth. This segment of the record will be modeled using the above analysis. The cone used for this test had a standard radius $a$ of $17.84 \mathrm{~mm}$ (giving a crosssectional area of $10 \mathrm{~cm}^{2}$ ). The raw value of $\bar{q}_{c}$ recorded in the soft soil was, on average, $0.8 \mathrm{MPa}$. This was converted into dimensionless form such that $\eta=4$ in the soft material, and $G_{1} \delta$ was set equal to

$$
G_{1} \delta=\frac{1}{4} \times 0.80 \times 17.84=3.58 \mathrm{MPa} \cdot \mathrm{mm}
$$

The positions of the two interfaces at top and bottom of the stiff layer were determined from the pore pressure record obtained simultaneously with the penetration record. Clear cut jumps in pore pressure occurred both at the top and bottom of the layer.

Based purely on the perceived values of $q_{\mathrm{c}}$ in the soft soil and in the layer, a value of $k_{1}$ equal to 5.4 was initially used to calculate the theoretical response. That calculation produced a theoretical curve which severely underestimated the resistance in the layer as shown in Figure 6. Returning to Figure 5 for a moment, one might suspect that the thin layer material could possibly be the same as that found above $8.5 \mathrm{~m}$ or below $10 \mathrm{~m}$, but that its perceived resistance is diminished due to its small layer thickness. The soils above $8.5 \mathrm{~m}$ and below $10 \mathrm{~m}$ both have mean penetration resistance roughly 15 times that found in the soft material. This suggests resetting $k_{1}$ to 15 and recalculating the theoretical response. When this is done the theoretical curve agrees with the field record much more closely as shown in Figure 6 . We are led to conclude that the thin layer at depth $9.4 \mathrm{~m}$ is in fact identical to the soils found above $8.5 \mathrm{~m}$ and below $10 \mathrm{~m}$. Clearly this conclusion would not be reached based on the raw data shown in Figure 5.

One final point can be made concerning Figure 6. In the figure, the location of the top of the layer appears to be slightly higher than the field resistance curve would suggest. This may be due to the fact that the standard procedure of referring all depths to the location of the cone tip has been followed. It may be that the experimental result should be compared with the analytical solution at the depth where the entire projected area of the cone is embedded, rather than at the tip. If allowance was made for this fact, the upper interface would be shifted downwards approximately $2 a$, and the analytical solution would fit the experimental data even more closely. Of course, the lower interface would also shift downward by the same amount, and this might cause the theoretical data to fit somewhat-less well at the base of the layer. It should be noted however that, at a dimensionless depth of $z / a=534$, the field penetration was halted for a brief time in order that a new drill rod be added to the string. This would have resulted in an unload/reload situation at the cone tip, and possibly distorted the experimental results near the base of the layer.

As a final example, let us consider how this correction for the layer effect might alter the results of liquefaction potential analyses which utilise CPT records. Figure 7 shows part of a

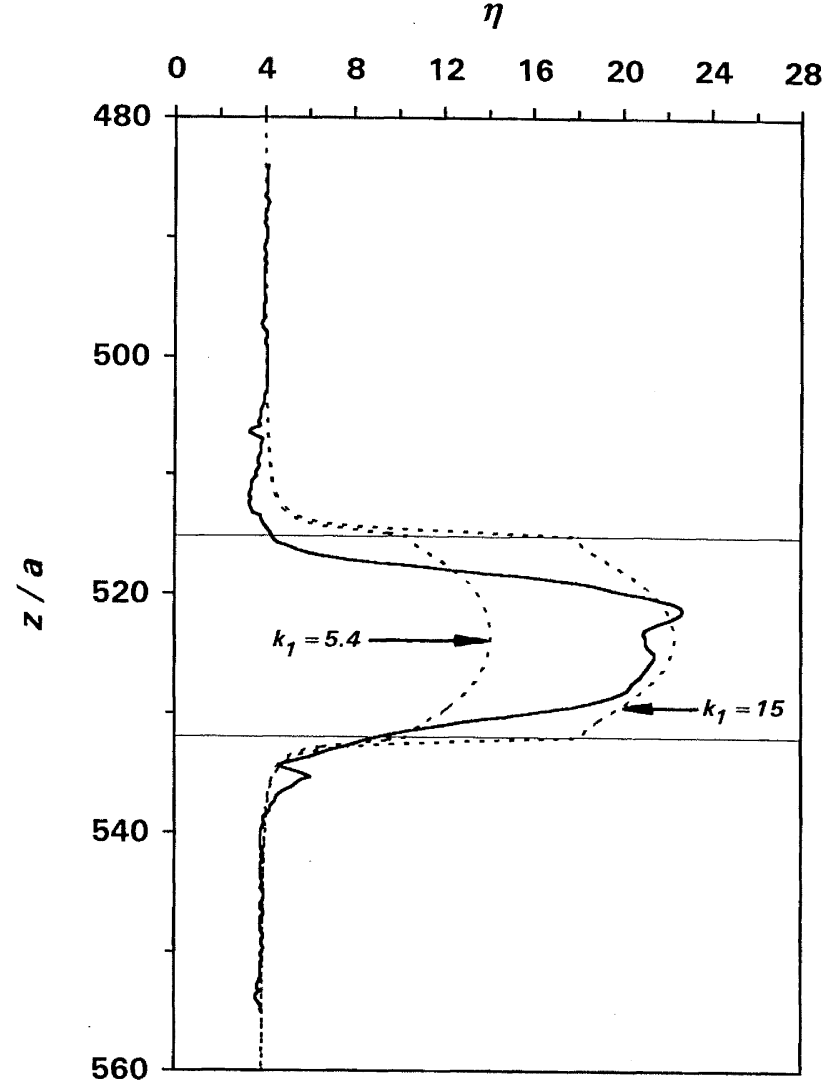

FIGURE 6. Enlargement from Figure 5 showing comparisons with theoretical response for two stiffness ratios.

CPTU record obtained at a site alongside the Pajaro River in central California, which liquefied during the Loma Prieta earthquake of October 1989.

Raw cone resistance, friction ratio and pore water pressure have been plotted, along with lines of critical $q_{c}$ evaluated using the following five models:

$$
\begin{aligned}
& \text { a - Taiping et al. [5] } \\
& \text { b - Davis \& Berrill [6] } \\
& \text { c - Shibata \& Teparaksa [7] } \\
& \text { d - Law, Cao \& He [8] } \\
& \text { e - Zhou [9] }
\end{aligned}
$$

These models predict the liquefaction potential of the soil by establishing a line of critical cone resistance. For a given seismic event and site, the soil is deemed likely to liquefy at depths where the measured cone resistance is less than the critical value. Note that the lines of critical $q_{c}$ plotted in Figure 7 are discontinuous. This results from the criterion of Robertson and Campanella [10], which has been applied so that the lines plot only when the $\left(q_{c}, R f\right)$ coordinate is within their proposed zone of liquefiable soil. In effect, plotting is turned off when the $\left(q_{c}, R f\right)$ point indicates a soil too fine to liquefy. The models make use of various earthquake source and site parameters to determine the line of critical cone resistance, including earthquake magnitude, Modified Mercalli Intensity, epicentral distance, mean grain size $\left(D_{50}\right)$, effective overburden stress and peak ground acceleration at the site under investigation. 

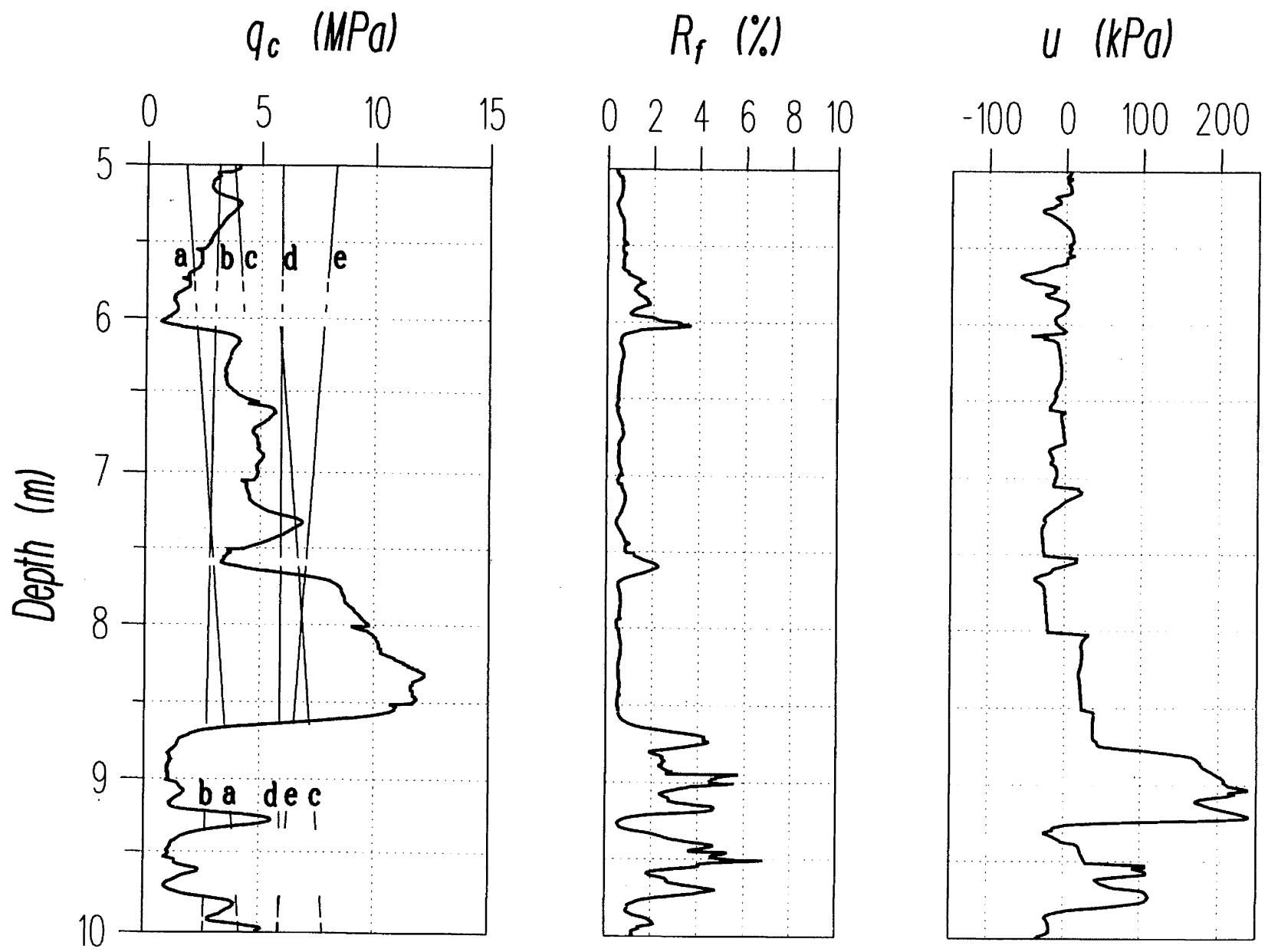

FIGURE 7. Section of a CPTU record at a site bordering the Pajaro River in California. Critical $q_{c}$ lines have been included from five liquefaction potential analyses.

It is evident from Figure 7 that all of the models predict that the soil between depths 5.75 and 6 metres is likely to have liquefied during the Loma Prieta earthquake. However, three of the models also predict liquefaction of a thin, stiff layer at a depth of 9.25 metres. We shall focus our attention on this layer, modelling the segment of the record between 9 and 9.5 metres in the same manner as in the previous example.

The CPTU was conducted using the same standard $10 \mathrm{~cm}^{2}$ cone. The raw $q_{c}$ values were converted to dimensionless cone resistance and scaled so that $\eta=4$ in the soil above the layer of interest by setting $G_{1} \delta$ equal to $6.37 \mathrm{MPa} \cdot \mathrm{mm}$. Once again, the pore water pressure record was used to determine the position of the upper and lower layer interfaces. This time, depths are referred to the shoulder of the cone rather than to the tip. The theoretical solution was calculated using an initial value of $k_{1}=3.9$, chosen on the basis of the perceived values of $q_{c}$ in the soft soil and in the thin, stiff layer. As before, this calculation severely underestimates the resistance in the layer as shown in Figure 8.

A second value of $k_{1}=8.4$ was then chosen, on the supposition that the thin layer might be of the same material as the stiff layer between 8 and 8.5 metres. This assumption is supported to some extent by the similarity of the $R f$ and $u$ records. The theoretical curve is shown in Figure 8. Although it matches the field record more closely, the resistance is still underestimated, and a better estimate could be obtained by further trial and error. Nevertheless, we are led to conclude that the thin layer soil is at least as stiff as the material between 8 and 8.5 metres, and that it is therefore unlikely to be susceptible to liquefaction.

It is important to note that in the context of the elastic analysis presented here, the layer of material between 7.5 and 8.5 metres also should be considered as "thin". Thus it is probable that the perceived resistance of this layer shown in Figure 7 is less than the resistance that would be recorded in a thick layer of the material at the same density. Hence it is still possible that the material of the two layers is the same.

Clearly, correction of this second profile is more difficult than the first, and makes apparent the need for a general inversion procedure for cone resistance recorded in multi-layered soil systems with arbitrary layer thickness and stiffness. Another problem raised by this analysis concerns the "average" layer thickness of data sets used in calibrating liquefaction models. A large number of calibration data from thin layers would have the effect of making a model unconservative when applied to thicker layers. 
$\eta$

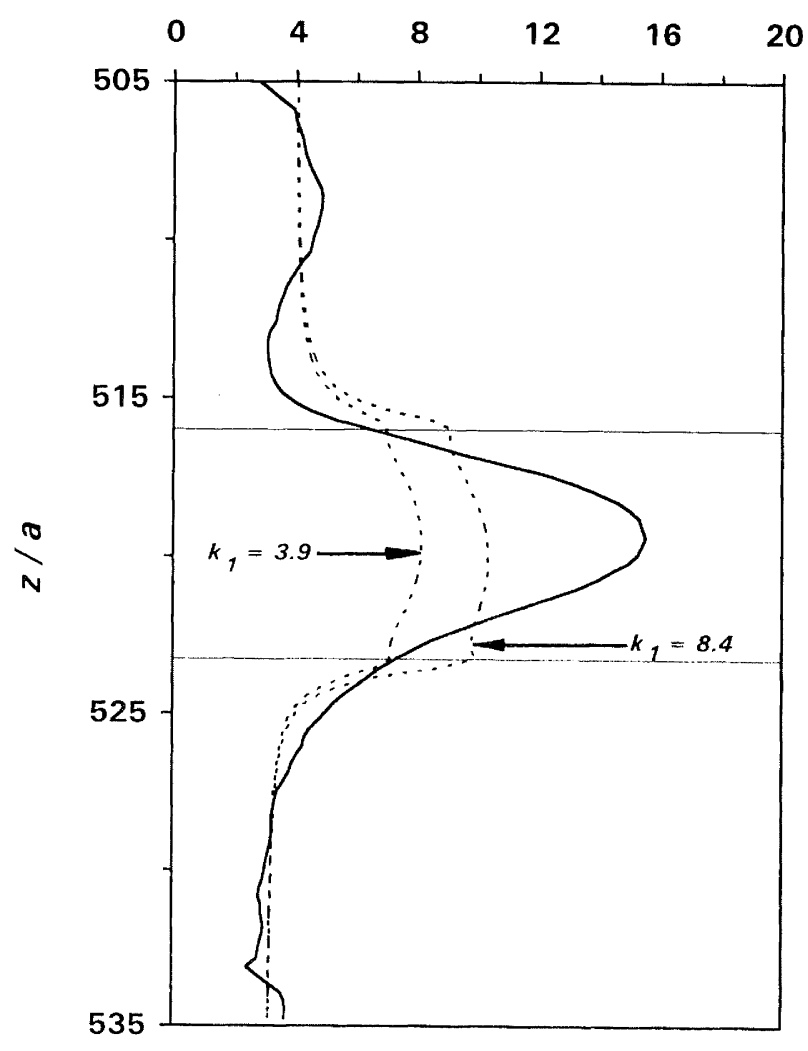

FIGURE 8. Enlargement from Figure 7 showing comparisons with theoretical response for two stiffness ratios.

\section{CONCLUSIONS}

In this paper a simple elastic analysis has been presented to explain how cone penetration resistance may be affected by the presence of nearby soil layers with different stiffness characteristics. It is suggested that the perceived penetration resistance may "sense" nearby layers. Comparisons with experimental data obtained in calibration chamber experiments and in the field have been presented which bear this out. The analysis has clear implications concerning the interpretation of cone data for soil classification, particularly where strength or liquefaction potential of thin layers happens to be under consideration. It is easily conceivable that a thin layer might be classified as susceptible to liquefaction based on perceived cone data, whereas, in fact, it is not. The simple elastic analysis presented here allows for a more reliable and realistic interpretation of any cone penetration data. Furthermore, layer thickness is clearly an important parameter to record in documenting case histories and to consider in devising and calibrating empirical models.

\section{ACKNOWLEDGEMENTS}

The authors wish to acknowledge the assistance of the New Zealand Earthquake and War Damage Commission (EQC) both for their direct support of the first author and for their general support of research into in situ testing of liquefaction sites. The calibration chamber results were obtained within the joint French-United States-New Zealand Projet Piézocône, funded principally by the French Ministry of Research and Technology, but also by the New Zealand Ministry of Energy, Transit New Zealand and the EQC. The Salinas and Pajaro River CPTU records result from a joint project with the United States Geological Survey, and the cooperation of the United States Geological Survey is gratefully acknowledged.

\section{REFERENCES}

1 Canou, J. 1989. Piézocône et liquéfaction des sables Rapport de synthèse des travaux réalisés au CERMES (19861988), Research Report, CERMES/ENPC, Paris, 176 pp.

2 Foray, P. \& J-L. Pautre. 1988. Piézocône et liquéfaction des sables: synthèse des essais sur sites en Nouvelle-Zélande et des essais en Chambre de Calibration à l'IMG, Research Report, IMG, Grenoble, 70 pp.

3 Plevako, V.P. 1969. A point force inside a pair of cohering half-spaces, Soil Mechanics and Foundation Eng., 6(3): 165-169.

4 Poulos, H.G. 1967. Stresses and displacements in an elastic layer underlain by a rough rigid base, Geotechnique, 17:378-410.

5 Taiping, Q., W. Chenchun, W. Lunian and L.Huishan. 1984. Liquefaction risk evaluation during earthquakes, Proc. Intl Conf. on Case Histories in Eng., St Louis, 1:445454.

6 Davis, R.O. \& J.B. Berrill. 1982. Energy dissipation and seismic liquefaction in sands, Earthquake Engineering and Structural Dynamics, 10:59-68.

7 Shibata, T. \& W. Teparaksa. 1988. Evaluation of liquefaction potentials of soils using cone penetration tests, Soils and Foundations, 28:49-60.

8 Law, K.T., Y.L. Cao \& G.N. He. 1990. An energy approach for assessing seismic liquefaction potential, Canadian Geotech. J., 27(3):320-329.

9 Zhou, S. 1980. Evaluation of the liquefaction of sand by static cone penetration test, Proc. 7th World Conf. Earthq. Eng., Istanbul, 3:156-162.

10 Robertson, P.K. \& R.G. Campanella. 1985. Liquefaction potential of sands using the CPT, J. Geotech. Eng., ASCE, 111:384-403 